\title{
Noncommutative Inspired Reissner-Nordström Black Holes in Large Extra Dimensions
}

\author{
Kourosh Nozari $^{a, b, *}$ and S. Hamid Mehdipour ${ }^{c, \dagger}$ \\ ${ }^{a}$ Department of Physics, Faculty of Basic Sciences, University of Mazandaran, \\ P. O. Box 47416-1467, Babolsar, IRAN \\ ${ }^{b}$ Research Institute for Astronomy and Astrophysics of Maragha, \\ P. O. Box 55134-441, Maragha, IRAN \\ ${ }^{c}$ Islamic Azad University, Lahijan Branch, \\ P. O. Box 1616, Lahijan, IRAN
}

\begin{abstract}
Recently, a new noncommutative geometry inspired solution of the coupled Einstein-Maxwell field equations including black holes in 4-dimension is found. In this paper, we generalize some aspects of this model to the Reissner-Nordström $(\mathrm{RN})$ like geometries with large extra dimensions. We discuss Hawking radiation process based on noncommutative inspired solutions. In this framework, existence of black hole remnant and possibility of its detection in LHC are investigated.
\end{abstract}

PACS: 04.70.-s, 04.70.Dy, 02.40.Gh, 04.50.+h

Key Words: Quantum Gravity, Black Hole Thermodynamics, Noncommutative Spacetime, Large Extra Dimensions

*knozari@umz.ac.ir

${ }^{\dagger}$ mehdipour@iau-lahijan.ac.ir 


\section{Introduction}

The underlying physics of black holes have been the target of many investigations. One of the important characteristic of a black hole is its thermodynamical properties: a black hole has Hawking temperature [1] which is proportional to its surface gravity on the horizon, and entropy of which is proportional to its horizon area [2]. These two quantities satisfy the first law of black hole thermodynamics. In this regard, studying thermal properties of various black holes is one of the significant subjects of black hole physics. Hawking has interpreted the quantum effect of black hole emission as a thermal radiant spectrum from event horizon, which sets a significant event in black hole physics. The discovery of this effect solved and revealed both the problem in black hole thermodynamics and the relation between quantum gravity and thermodynamics. Hawking has pointed out that when the virtual particles near the surface of the black hole with negative energy come into black hole via tunnel effect, the energy of the black hole will decrease and the radius of the black hole event horizon will decrease also. This process is equivalent to the emission of a particle from the black hole (black hole evaporation). But, how is the final stage of black hole evaporation? The final stage of the black hole evaporation is a matter of debates in the existing literature [3]. The generalized uncertainty principle (GUP), motivated by string theory and noncommutative quantum mechanics, suggests significant modifications to the Hawking temperature and evaporation process of black holes. Adler et al [4] have argued that contrary to standard view point, GUP may prevent small black holes from total evaporation in exactly the same manner that the usual uncertainty principle prevents the Hydrogen atom from total collapse.

Nicolini, Smailagic and Spallucci (NSS) [5] have found a noncommutative geometry inspired solution of the Einstein equation smoothly interpolating between a de Sitter core around the origin and an ordinary Schwarzschild spacetime at large distances. Many studies have been performed in these directions where spacetime is commutative. Noncommutative spacetime view point [6], gets special appeal due to telling beforehand of string theory, leads to the fact that spacetime points might be noncommutative. Undoubtedly, spacetime noncommutativity can cure some kind of divergences, which appear in General Relativity. The inclusion of noncommutativity in black hole metric has been studied in $[7,8]$. It has been shown that the modified metric due to noncommutativity of spacetime does not allow the black hole to decay beyond a minimal mass $M_{0}$. Then, the evaporation process terminates when black hole reaches a Planck size remnant with zero temperature, which does not diverge at all, rather it reaches a maximum value before cooling down to absolute zero.

The authors in Ref. [9] have generalized the NSS model to the case where flat, toroidally compactified extra dimensions are accessible at the $T e V$ energy scale. During the last 
decade, several models using compactified large extra dimensions (LEDs) scenarios $[10,11]$ have been proposed, which have significant implications for processes involving strong gravitational fields, such as the decay of black holes. In models with extra spatial dimensions the four dimensional spacetime is viewed as a $D_{3}$-brane embedded in a bulk spacetime of dimension $d$, (where $d \geq 4$ ). Embedding a black hole in a spacetime with additional dimensions would seem, from the string theory point of view, to be a natural thing to do. For extra-dimensional gravity with $\mathrm{TeV}$ energy scales, Hawking temperature and evaporation process of black holes lead to important changes in the formation and detection of black holes at the Large Hadronic Collider (LHC) [13]. Since a black hole can evaporate into all existing particles whose masses are lower than its temperature, thus these fascinating processes could be tested at the LHC, and providing a way of testing the existence of extra dimensions.

Recently, Ansoldi, Nicolini, Smailagic and Spallucci (ANSS) [14] along their previous studies, have found a new, noncommutative inspired solution of the coupled Einstein-Maxwell field equations including black holes in 4-dimensional brane universe. In this paper we are going to generalize their model to large extra dimensions scenario. So, the main purpose of this paper is to consider the effect of space noncommutativity on the short distance thermodynamics of an evaporating RN black hole in $d$-dimensional spacetime. We investi-

gate the possibility of formation of black holes remnants and we discuss the energy scales for detection of these remnants at LHC. We also discuss the evidences for non-extensive thermodynamics of such a short distance systems.

The layout of the paper is as follows: we begin in Section 2 by outlining the RN black holes in spacetime dimensions higher than four and their generalizations to a regular de Sitter vacuum accounting for the effect of noncommutative coordinate fluctuations at short distances (noncommutative inspired $\mathrm{RN}$-dS solutions) in $d$-dimensional bulk spacetime. In Section 3 we pay special attention to the thermodynamic behavior of RN-dS black holes by study of Hawking temperature, entropy, specific heat and free energy in various dimensions. The paper follows by summary and discussion in Section 4 .

\section{Noncommutative Inspired Charged Black Holes in Large Extra Dimensions}

The RN black hole is a solution of the Einstein equation coupled to the Maxwell field. The classical RN metric is

$$
d s^{2}=\frac{\Delta}{r^{2}} d t^{2}-\frac{r^{2}}{\Delta} d r^{2}-r^{2} d \Omega_{2}^{2},
$$


where $d \Omega_{2}^{2}$ is the metric on the unit $S^{2}$ and

$$
\Delta \equiv r^{2}-2 M r+Q^{2} \equiv\left(r-r_{+}\right)\left(r-r_{-}\right),
$$

with

$$
r_{ \pm}=M \pm \sqrt{M^{2}-Q^{2}}
$$

Let us now consider the charged black hole thermodynamics in model universes with large extra dimensions. There are two main scenarios of large extra dimensions (LEDs)

- the Arkani-Hamed-Dimopoulos-Dvali (ADD) model [10], where the extra dimensions are compactified toroidally and all of radius $R$. This model was motivated by the desire to provide a solution to the so-called hierarchy problem, that is, the sixteen orders of magnitude difference between the electroweak energy scale and the Planck scale;

and

- the Randall-Sundrum (RS) model [11], where the extra dimensions have an infinite extension but are warped by a non-vanishing cosmological constant. This model also solve the hierarchy problem despite a different approach to the ADD model.

In LEDs scenario, RN metric can be written as follows

$$
d s^{2}=\left(1-\frac{2 m}{r^{d-3}}+\frac{q^{2}}{r^{2(d-3)}}\right) d t^{2}-\left(1-\frac{2 m}{r^{d-3}}+\frac{q^{2}}{r^{2(d-3)}}\right)^{-1} d r^{2}-r^{2} d \Omega_{(d-2)}^{2},
$$

where $d \Omega_{(d-2)}^{2}$ is the line element on the $(d-2)$-dimensional unit sphere and $d$ is spacetime dimensionality. The volume of the $(d-2)$-dimensional unit sphere is given by

$$
\Omega_{(d-2)}=\frac{2 \pi^{\frac{d-1}{2}}}{\Gamma\left(\frac{d-1}{2}\right)} .
$$

$g_{00}$ is a function of mass and charge given in terms of parameters $m$ and $q$ as follows

$$
m=\frac{8 \pi G_{d}}{(d-2) \Omega_{(d-2)}} M,
$$

and

$$
q=\sqrt{\frac{8 \pi G_{d}}{(d-2)(d-3)}} Q .
$$

$G_{d}$ is gravitational constant in $d$-dimensional spacetime which in ADD model is given by

$$
G_{d}=\frac{(2 \pi)^{d-4}}{\Omega_{d-2}} M_{P l}^{2-d},
$$

\footnotetext{
${ }_{\ddagger}^{\ddagger}$ The model proposed by Dvali, Gabadadze and Porrati (DGP) [12] is essentially different with above mentioned scenarios since it predicts deviations from the standard 4-dimensional gravity even over large distances. However, in this paper we restrict our study to the ADD model.
} 
where $M_{P l}$ is the $d$-dimensional Planck mass and there is an effective 4-dimensional Newton constant related to $M_{P l}$ by

$$
M_{P l}^{2-d}=4 \pi G_{4} R^{d-4}
$$

where $R$ is the size of extra dimensions. It is necessary to note that in this work, the conventions for definition of the fundamental Planck scale $M_{P l}$ are the same as which have been used by ADD and also GT [15]. ( Hereafter we set the fundamental constants equal to unity; $\hbar=c=k_{B}=4 \pi \epsilon_{0}=1$ ). In this section, we will obtain and investigate the noncommutative inspired RN solution for a black hole in large extra dimensions, where noncommutativity can be taken as the correction to the RN black hole metric and goes to zero when the strength of noncommutativity goes to zero. The simplest noncommutativity that one can postulate is the commutation relation $\left[\mathbf{x}^{\mathbf{i}}, \mathbf{x}^{\mathbf{j}}\right]=\mathbf{i} \theta^{\mathbf{i j}}$, with a parameter $\theta$ which measures the amount of coordinate noncommutativity in the coordinate coherent states approach [16] and $\theta^{i j}$ is an antisymmetric (constant) tensor of dimension $(\text { length })^{2}$.

The approach we adopt here is to look for a static, asymptotically flat, spherically symmetric, minimal width, Gaussian distribution of mass and charge whose noncommutative size is determined by the parameter $\sqrt{\theta}$. To do this end, we shall model the mass and charge distributions by a smeared delta function $\rho([5,9,14])$

$$
\left\{\begin{array}{l}
\rho_{\text {matt }}(r)=\frac{M}{(4 \pi \theta)^{\frac{d-1}{2}}} e^{-\frac{r^{2}}{4 \theta}} \\
\rho_{e l}(r)=\frac{Q}{(4 \pi \theta)^{\frac{d-1}{2}}} e^{-\frac{r^{2}}{4 \theta}}
\end{array}\right.
$$

The assumption of spherical symmetry means that the line element reduces to the canonical form, namely,

$$
d s^{2}=e^{\nu} d x_{0}^{2}-e^{\mu} d r^{2}-r^{2} d \Omega_{d-2}^{2}
$$

and

$$
d \Omega_{d-2}^{2}=d \vartheta_{d-3}^{2}+\sin ^{2} \vartheta_{d-3}\left(d \vartheta_{d-4}^{2}+\sin ^{2} \vartheta_{d-4}\left(\ldots+\sin ^{2} \vartheta_{2}\left(d \vartheta_{1}^{2}+\sin ^{2} \vartheta_{1} d \varphi^{2}\right) \ldots\right)\right),
$$

where $0<\varphi<2 \pi$ and $0<\vartheta_{i}<\pi$, for $i=1, \ldots, d-3$. In the above formulae, $\nu$ and $\mu$ are functions of $r$ only, because we impose the condition that the solution is static and our assumption that the solution is asymptotically flat requires: $\nu, \mu \rightarrow 0$ as $r \rightarrow \infty$; this will require that $\nu=-\mu$ in the solutions of Einstein-Maxwell field equations.

The system of Einstein-Maxwell field equations is as follows

$$
\left\{\begin{array}{l}
R_{A}^{B}-\frac{1}{2} \delta_{A}^{B} R=8 \pi G_{d}\left(\left.T^{B}{ }_{A}\right|_{\text {matt }}+\left.T^{B}{ }_{A}\right|_{e l}\right) \\
\frac{1}{\sqrt{-g}} \partial_{B}\left(\sqrt{-g} F^{B A}\right)=J^{A}
\end{array}\right.
$$


where $\left.T_{A}^{B}\right|_{\text {matt }}=\operatorname{diagonal}\left(-\rho_{\text {matt }}(r), p_{r}, p_{\vartheta_{1}}, \ldots, p_{\vartheta_{d-3}}, p_{\phi}\right)$, are comprised of a radial pressure $p_{r}=-\rho_{\text {matt }}(r)$ and tangential pressure of a self-gravitating anisotropic fluid $p_{\vartheta_{i}}=p_{\phi}=-\rho_{\text {matt }}(r)-\frac{r}{(d-2)} \partial_{r} \rho_{\text {matt }}(r)$, while the electromagnetic energy-momentum tensor must take on the form

$$
F^{B A}=\delta^{0[B \mid} \delta^{r \mid A]} E_{d}(r ; \theta)=E_{d}(r ; \theta)\left(\begin{array}{ccccc}
0 & -1 & 0 & \ldots & 0 \\
1 & 0 & 0 & \ldots & 0 \\
0 & 0 & 0 & \ldots & 0 \\
\vdots & \vdots & \vdots & \ddots & \vdots \\
0 & 0 & 0 & \ldots & 0
\end{array}\right),
$$

where smearing of the electric field reads

$$
E_{d}(r ; \theta)=\frac{Q}{r^{2(d-3)}} \frac{\gamma\left(\frac{d-1}{2}, \frac{r^{2}}{4 \theta}\right)}{\Gamma\left(\frac{d-1}{2}\right)} .
$$

Then the Einstein field equations $G_{B A}=8 \pi G_{d} T_{B A}$ lead to the following solution

$$
d s^{2} \equiv g_{B A} d x^{B} d x^{A}=g_{00} d t^{2}-g_{00}^{-1} d r^{2}-r^{2} d \Omega_{(d-2)}^{2},
$$

with

$$
\left\{\begin{array}{l}
g_{00}=1-\frac{2 m}{r^{d-3}} \frac{1}{\Gamma\left(\frac{d-1}{2}\right)} \gamma\left(\frac{d-1}{2}, \frac{r^{2}}{4 \theta}\right)+\frac{(d-3)^{2}(d-2)}{2 \pi^{d-3}} \frac{q^{2}}{r^{2(d-3)}} F(r) \\
F(r)=\gamma^{2}\left(\frac{d-3}{2}, \frac{r^{2}}{4 \theta}\right)-\frac{2^{\frac{11-3 d}{2}}}{(d-3) \theta^{\frac{d-3}{2}}} \gamma\left(\frac{d-3}{2}, \frac{r^{2}}{4 \theta}\right) r^{d-3} \\
\gamma\left(\frac{a}{b}, u\right)=\int_{0}^{u} \frac{d t}{t} t^{\frac{a}{b}} e^{-t} .
\end{array}\right.
$$

In fact, by plugging the above metric into the Einstein-Maxwell system, the $g_{00}$ can be determined, although it is done slightly simpler, for the larger values of $d$ with a good approximation, by plugging the explicit form for the smeared mass and charge distributions into the metric as follows

$$
g_{00}=1-\frac{2 m_{\theta}}{r^{d-3}}+\frac{q_{\theta}^{2}}{r^{2(d-3)}},
$$

with

$$
\left\{\begin{array}{l}
m_{\theta}=\frac{8 \pi G_{d}}{(d-2) \Omega_{(d-2)}} M_{\theta} \\
q_{\theta}=\sqrt{\frac{8 \pi G_{d}}{(d-2)(d-3)}} Q_{\theta},
\end{array}\right.
$$

where $M_{\theta}$ and $Q_{\theta}$ are the smeared mass and charge distributions respectively and are decided by

$$
\left\{\begin{array}{l}
M_{\theta}=\int_{0}^{r} \rho_{\text {matt }}(r) \Omega_{(d-2)} r^{2} d r=\frac{\gamma\left(\frac{d-1}{2}, \frac{r^{2}}{4 \theta}\right)}{\Gamma\left(\frac{d-1}{2}\right)} M \\
Q_{\theta}=\int_{0}^{r} \rho_{e l}(r) \Omega_{(d-2)} r^{2} d r=\frac{\gamma\left(\frac{d-1}{2}, \frac{r^{2}}{4 \theta}\right)}{\Gamma\left(\frac{d-1}{2}\right)} Q
\end{array}\right.
$$


The above metric smoothly interpolates between de Sitter core around the origin and an ordinary Reissner-Nordström geometry far away from the origin (RN-dS Black Hole in large extra dimensions). On the other hand, the curvature singularity at $r=0$ is eliminated by noncommutativity as an intrinsic property of the manifold. In this situation, a regular de Sitter vacuum state will be formed accounting for the effect of noncommutative coordinate fluctuations at short distances and also a usual Reissner-Nordström spacetime at large distances is being taken into account in higher than 4-dimension. Classical RN metric for large distances in 4-dimension is also obtained from (13) in the limit of $\theta \rightarrow 0$ or $r \gg \theta$ and $d=4$.

The event horizon radius, $r_{H}$, can be obtained from the equation $g_{00}\left(r_{H}\right)=0$ which gives

$$
1-\frac{2 m_{\theta}}{r_{H}^{d-3}}+\frac{q_{\theta}^{2}}{r_{H}^{2(d-3)}}=0 .
$$

Depending on the different values of $Q, M$ and $M_{P l}$, the metric displays three possible causal structure $[5,9,14]$ : $\mathbf{1}$ - It is possible to have two distinct horizons (Non-extremal Black Hole), 2- It is possible to have one degenerate horizon (Extremal Black Hole), and finally 3- It is impossible to have horizon at all (Massive Charged Droplet).

It is important to note that, the $d$-dimensional Planck mass $M_{P l}$ in LEDs models might be as low as a $T e V$-scale, because it is found that, this $T e V$-scale is very directly constrained by experimental bounds and it is also required to be $\sim 1 \mathrm{TeV}$ in order to solve the hierarchy problem, which is relevant for black hole production at near-future experiments (LHC and also in ultra high energy cosmic ray showers [17]). Consequently, the minimum energy for possible formation and detection of black holes at LHC is decreased, if $M_{P l} \sim 1 \mathrm{TeV}$. Indeed, the minimal mass of black hole depends sensitively on the fundamental Planck scale, $M_{P l}$, and on the spacetime dimension, $d$. Based on this feature, in the following figures, 1 and 2, the fundamental Planck mass has been set equal to $M_{P l}=0.5 \mathrm{TeV}$, while in figure 3 we have set $M_{P l}=1.5 \mathrm{TeV}$. In all of these figures, the initial mass of black hole has been chosen to be $M=5 \mathrm{TeV}$. These figures show that, if the initial mass of black hole as energy scales accessible at the LHC is not large enough, then LHC will not see any black hole in this regime. Also, figure 2 shows that possibility of black hole formation is reduced by increasing the charge of black hole particularly for 4-dimensional black hole on the brane.

Analytical solution of equation (14) for $r_{H}$ in a closed form is impossible, so we solve it numerically to find this quantity. However, it is possible to solve (14) to find $M$, which provides the mass as a function of the horizon radius $r_{H}$ and charge $Q$ in an arbitrary dimension. If we have chosen a finite dimension (for example $d=4, d=5$ and so on), then the mass of RN-dS black hole as a function of the horizon radius and charge can be 


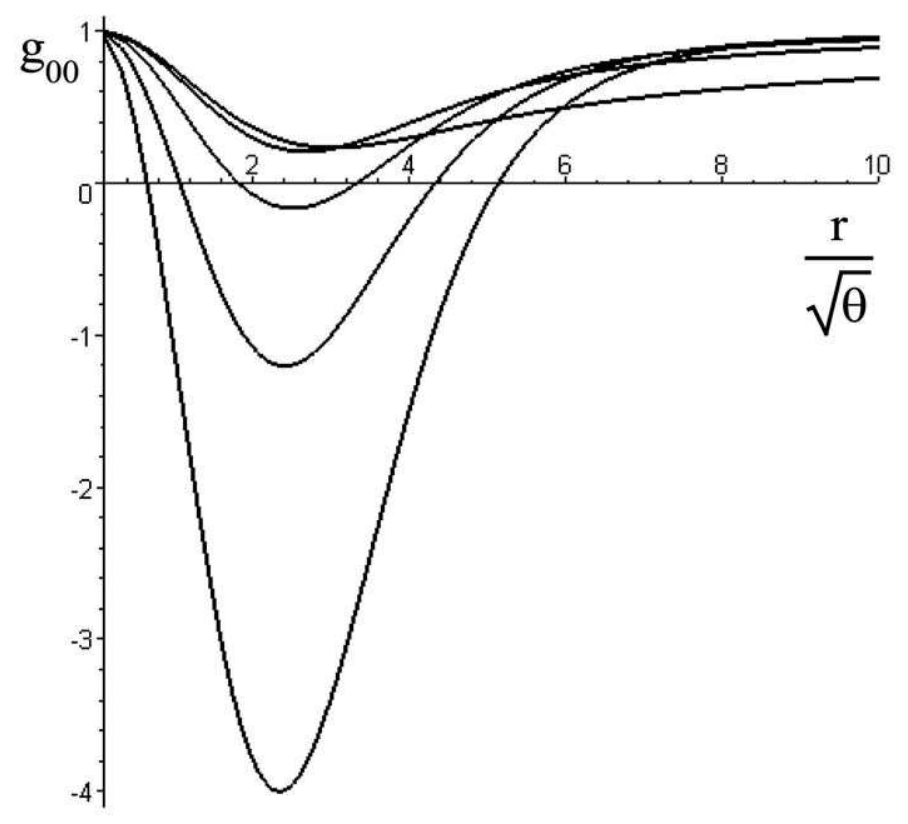

Figure 1: $g_{00}$ versus the radius $r$ in $\sqrt{\theta}$ units for different dimensions. Black hole charge, mass and $d$-dimensional Planck mass are set equal to $Q=0.5, M=5$ and $M_{P l}=0.5$ respectively. On the left-hand side of the figure, curves are marked from top to bottom by $d=4$ to $d=8$. This figure shows the possibility of having extremal configuration by decreasing the number of spacetime dimensions.

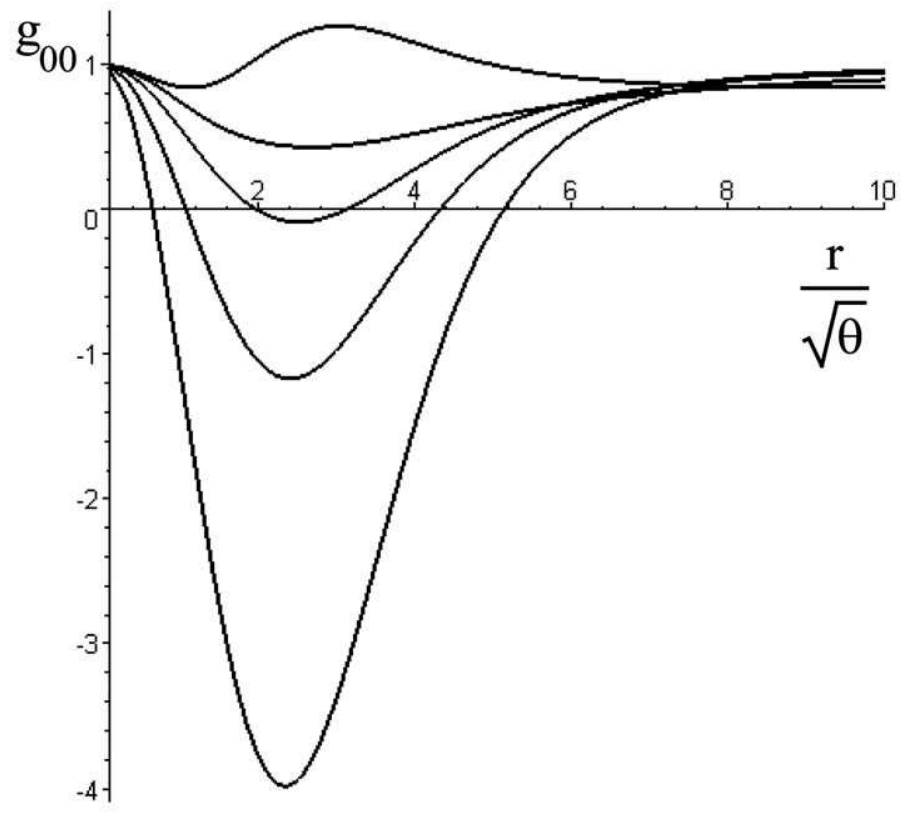

Figure 2: $g_{00}$ versus the radius $r$ in $\sqrt{\theta}$ units for different number of dimensions. Black hole charge and mass and $d$-dimensional Planck mass are set equal to $Q=2, M=5$ and $M_{P l}=0.5$ respectively. On the left-hand side of the figure, curves are marked from top to bottom by $d=4$ to $d=8$. This figure is the same as previous one: possibility of extremal configuration by decreasing the number of spacetime dimensions. However, in comparison with previous figure, we see a significant difference for black hole on the 3-brane when the charge varies. This may be a reflection of the fact that black hole lives on the brane and radiates mainly on the brane [18]. 


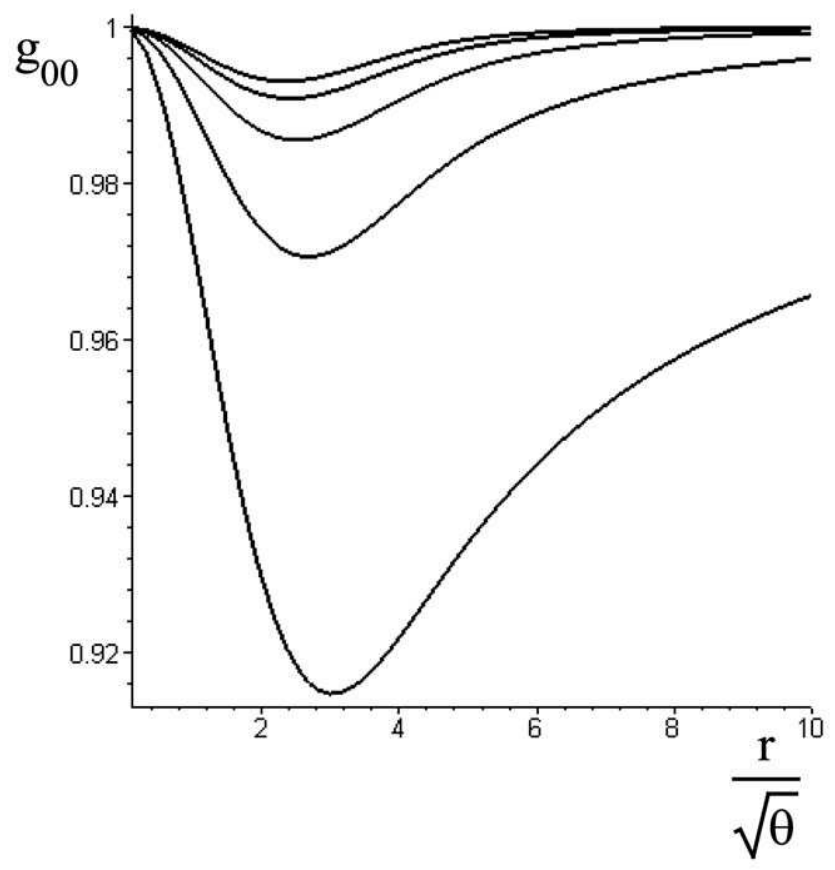

Figure 3: $g_{00}$ versus the radius, $r$ in $\sqrt{\theta}$ units for different number of spacetime dimensions. Black hole charge and mass and the $d$-dimensional Planck mass are set equal to $Q=0.5, M=5$ and $M_{P l}=1.5$ respectively. On the left-hand side of the figure, curves are marked from bottom to top by $d=4$ to $d=8$. The figure shows that in this case there is no horizon and then no black hole is formed.

obtained by solving equation (14). This leads us to

$$
\begin{gathered}
\left.d=4 \Longrightarrow M=\frac{\sqrt{\pi} r_{H}^{2} \theta+4 G_{4} Q^{2}\left(\pi^{\frac{3}{2}} \theta \mathcal{E}\left(\frac{r_{H}}{\sqrt{2 \theta}}\right)^{2} e^{\frac{r_{H}^{2}}{4 \theta}}+\sqrt{\pi} r_{H}^{2} e^{-\frac{r_{H}^{2}}{4 \theta}}-\frac{2 \pi r_{H}}{\sqrt{\theta}} \mathcal{E}\left(\frac{r_{H}}{\sqrt{2 \theta}}\right)\right)}{-2 G_{4} r_{H}^{2} \theta^{\frac{1}{2}}+2 G_{4} r_{H} \theta \sqrt{\pi} \mathcal{E}\left(\frac{r_{H}}{\sqrt{2 \theta}}\right) e^{\frac{r_{H}^{2}}{4 \theta}}}, \quad 15\right) \\
d=5 \Longrightarrow M=\frac{-\frac{3}{2} \pi r_{H}^{4} \theta^{2}-\pi^{2} G_{5} e^{-\frac{r_{H}^{2}}{2 \theta}} Q^{2}\left(\frac{r_{H}^{4}}{8}+r_{H}^{2} \theta-r_{H}^{2} \theta e^{\frac{r_{H}^{2}}{4 \theta}}+2 \theta^{2}-4 \theta^{2} e^{\frac{r_{H}^{2}}{4 \theta}}+2 \theta^{2} e^{\frac{r_{H}^{2}}{2 \theta}}\right)}{G_{5} r_{H}^{4} \theta e^{-\frac{r_{H}^{2}}{4 \theta}}+4 G_{5} r_{H}^{2} \theta^{2} e^{-\frac{r_{H}^{2}}{4 \theta}}-4 G_{5} r_{H}^{2} \theta^{2}},
\end{gathered}
$$

and so on. $\mathcal{E}(x)$ shows the Gauss Error Function defined as follows

$$
\mathcal{E}(x) \equiv \frac{2}{\sqrt{\pi}} \int_{0}^{x} e^{-t^{2}} d t
$$

When $d$ is even, we see that these equations can be expressed in terms of combinations of error functions. When $d$ is odd, it is possible to solve these equations analytically. The results of numerical solution of the mass of RN-dS black hole as a function of the horizon radius are presented in figures 4 and 5. As these two figures show, assuming a small enough $M_{P l}$, it is possible to detect the $T e V$ black holes at the expected LHC-energies. The center of mass energy of the proton-proton $(p p)$ collision at LHC lab is $14 T e V$. In this noncommutative framework black hole formation is possible only for some minimum mass of $M_{\min }<14 \mathrm{TeV}$. This is a pure noncommutative effect. In commutative case this 


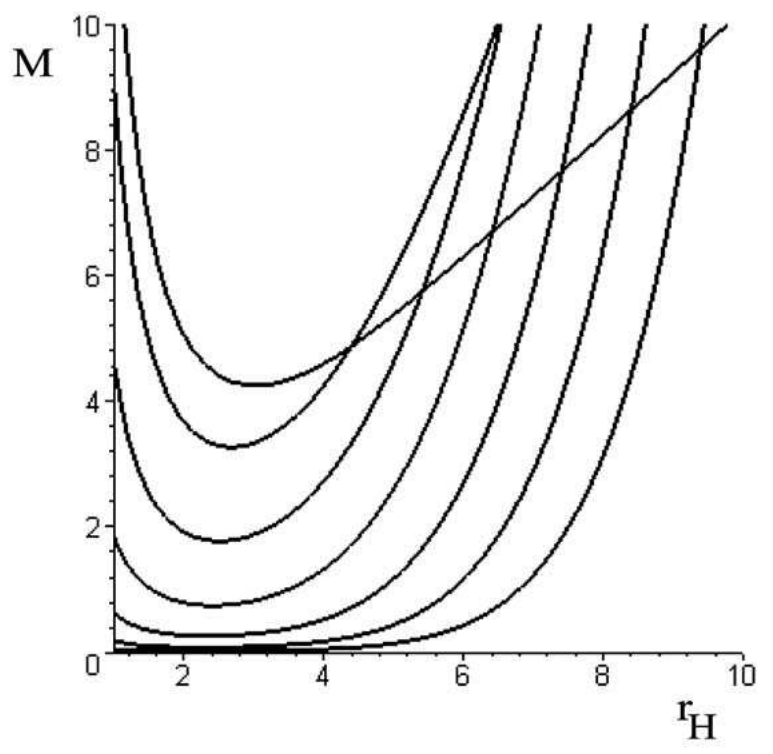

Figure 4: Black hole mass $M$ versus the radius of event horizon, $r_{H}$, for different number of spacetime dimensions. Black hole charge and the $d$-dimensional Planck mass are set equal to $Q=0.5$ and $M_{P l}=0.4$ respectively. On the left-hand side of the figure, curves are marked from top to bottom by $d=4$ to $d=10$. Since the center of mass energy of the proton-proton collision at LHC is $14 \mathrm{TeV}$, black hole formation is possible for $M_{\min }<14 \mathrm{TeV}$. So this figure shows the possibility of formation and detection of $\mathrm{TeV}$ black hole at the LHC.

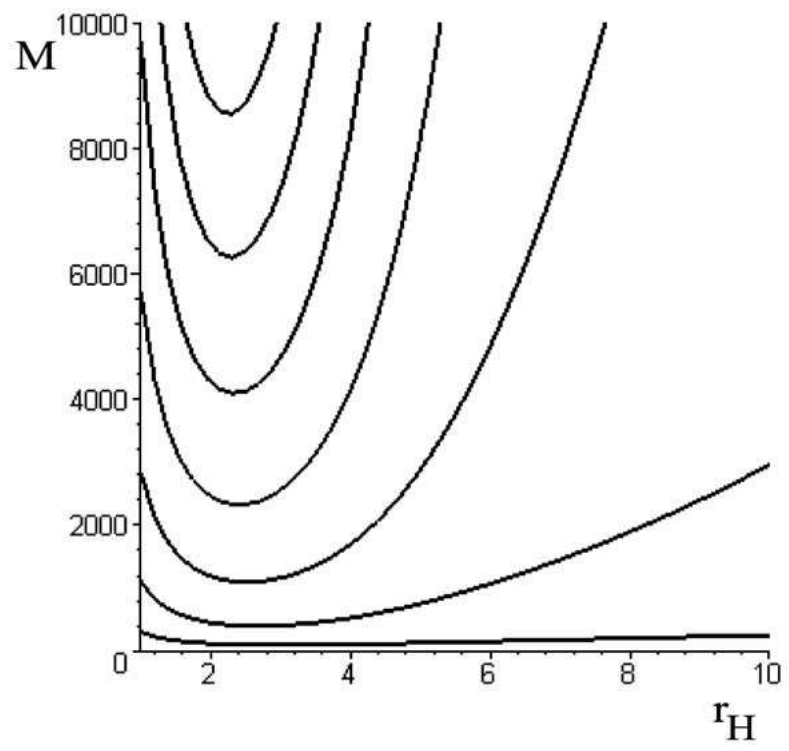

Figure 5: Black hole mass $M$ versus the radius of event horizon $r_{H}$ for different number of spacetime dimensions. Black hole charge and the $d$-dimensional Planck mass are set equal to be $Q=0.5$ and $M_{P l}=2$ respectively. On the right-hand side of the figure, curves are marked from bottom to top by $d=4$ to $d=10$. The figure shows that in this case there is no black hole in the energy scales accessible at the LHC. 
minimum value reduces to zero. As figure 4 (with $M_{P l}=0.4 \mathrm{TeV}$ and spacetime dimension $d=6$ ) shows, the minimum black hole mass in this situation is $1.8 \mathrm{TeV}$ approximately. In figure 5 , which is determined with $M_{P l}=2 \mathrm{TeV}$ and $d=6$, the minimum mass of the black hole is going to be about $1100 \mathrm{TeV}$ which is impossible to be formed in LHC. Hence, the possibility of forming these $10^{3} \mathrm{TeV}$ black holes at the LHC shrink to zero, however it is possible to be formed in the ultrahigh energy cosmic ray (UHECR) airshowers [17]. Also, figures 4 and 5 show that, if the number of spacetime dimension increases at a small enough $M_{P l}$, then the probability of forming and producing black hole at the LHC will increase. On the other hand, in this situation the minimal black hole mass threshold for producing and detecting black hole at the LHC reduces. Contrary to this, if the number of spacetime dimension, $d$, increases with a larger amount of $d$-dimensional Planck mass, then the minimum energy for black hole formation in collisions will increase and we will not see any black hole at the usual $\mathrm{TeV}$ energy scales.

The metric (12) shows a meaningful asymptotic behavior at short distances. By using the asymptotic form of the metric (12), we find the de Sitter type solutions with line element such that

$$
g_{00}=1-\frac{c_{d} M G_{d}}{\pi^{\left(\frac{d-3}{2}\right)} \theta^{\left(\frac{d-1}{2}\right)}} r^{2}+O\left(r^{3}\right),
$$

where $c_{d}$ is a dimensionless numerical constant which depends on the number of spacetime dimensions. Since the physical effect of noncommutativity is realized by substituting the position Dirac-delta corresponding to point-like profiles with Gaussian function of minimal width $\sqrt{\theta}$ describing the corresponding smeared profiles $[5,9,14,16,19]$, this form of structure has a regular de Sitter vacuum solution accounting for the effect of noncommutative coordinate fluctuations at short distances. The effective density of vacuum energy corresponds to the effective cosmological constant,

$$
\Lambda_{e f f}=\frac{c_{d} M G_{d}}{\pi^{\left(\frac{d-3}{2}\right)} \theta^{\left(\frac{d-1}{2}\right)}},
$$

which is leading to a finite curvature in the origin. It is interesting to see that there is no charge term in the effective cosmological constant. This is due to the fact that the electric field has linear behavior at short distances [14], which can only give raise to charge term of order $O\left(r^{3}\right)$ in the metric. Thus, an observer close to the origin sees only a vacant mass $M$ without any charge contribution.

It is believed that noncommutativity can cure divergences that appear, under the variety of forms, in General Relativity. For instance, it would be of special interest to investigate the final stage of black hole evaporation and some related thermodynamical quantities of black hole in the framework of noncommutative coordinates. In the next section we study this issue with details. 


\section{Thermodynamics of Noncommutative RN-dS Black Holes}

Black hole thermodynamics has continued to fascinate researchers since Hawking's discovery of the thermal radiation from black holes, because it prepares a real connection between gravity and quantum mechanics. The study of black hole thermodynamics also played a crucial role in the extension of quantum field theory in curved spacetime [20,21]. Hawking radiation shows how quantum fields on black hole backgrounds behave thermally. In this regard, black hole evaporation due to Hawking radiation is one of the fascinating dynamical behaviors of a black hole structure. Although black holes are perhaps the most perfectly thermal objects in the universe, but their thermal properties are not fully understood yet. This section aims to analyze some thermodynamical properties of the RN-dS black hole and some problems about the final stage of black hole evaporation in $d$ dimension with the hope that a little progress in this direction to be achieved. Therefore, our next step is to determine the thermodynamic behavior of noncommutative inspired RN-dS black holes. To do this end, we should calculate Hawking temperature of the black hole. The Hawking temperature can be obtained in the usual manner by remembering that

$$
T_{H}=\left.\frac{1}{4 \pi} \frac{d g_{00}}{d r}\right|_{r=r_{+}} .
$$

When $d$ is odd, we can solve this equation analytically, however for even $d$, it is impossible to solve it analytically and we must perform numerical calculation of Hawking temperature. Black hole temperature with some odd number of dimensions can be calculated as follows

$$
\begin{gathered}
d=5 \rightarrow T_{H}=\frac{1}{4 \pi}\left(-M G_{5}\left[\frac{r_{+}}{3 \pi \theta^{2}}+\frac{4 X_{5}}{3 \pi \theta r_{+}^{3}}\right]+Q^{2} G_{5}\left[-\frac{\pi X_{5} e^{-\frac{r_{+}^{2}}{4 \theta}}}{12 \theta^{3} r_{+}}-\frac{\pi X_{5}^{2}}{3 \theta^{2} r_{+}^{5}}\right]\right), \\
d=7 \rightarrow T_{H}=\frac{1}{4 \pi}\left(-M G_{7}\left[\frac{r_{+} e^{-\frac{r_{+}^{2}}{4 \theta}}}{20 \pi^{2} \theta^{3}}+\frac{2 X_{7}}{5 \pi^{2} \theta^{2} r_{+}^{5}}\right]+Q^{2} G_{7}\left[-\frac{\pi X_{7} e^{-\frac{r_{+}^{2}}{4 \theta}}}{2560 \theta^{5} r_{+}^{3}}-\frac{\pi X_{7}^{2}}{320 \theta^{4} r_{+}^{9}}\right]\right),(21) \\
d=9 \rightarrow T_{H}=\frac{1}{4 \pi}\left(-M G_{9}\left[\frac{r_{+} e^{-\frac{r_{+}^{2}}{4 \theta}}}{112 \pi^{3} \theta^{4}}+\frac{3 X_{9}}{28 \pi^{3} \theta^{3} r_{+}^{7}}\right]+Q^{2} G_{9}\left[-\frac{\pi X_{9} e^{-\frac{r_{+}^{2}}{4 \theta}}}{774144 \theta^{7} r_{+}^{5}}-\frac{\pi X_{9}^{2}}{64512 \theta^{6} r_{+}^{13}}\right]\right),
\end{gathered}
$$

and so on. $\quad X_{5}, X_{7}$, and $X_{9}$ are functions of $r_{+}$and $\theta$ defined as follows

$$
X_{5}=e^{-\frac{r_{+}^{2}}{4 \theta}}\left(r_{+}^{2}+4 \theta-4 \theta e^{\frac{r_{+}^{2}}{4 \theta}}\right),
$$




$$
\begin{gathered}
X_{7}=e^{-\frac{r_{+}^{2}}{4 \theta}}\left(r_{+}^{4}+8 \theta r_{+}^{2}+32 \theta^{2}-32 \theta^{2} e^{\frac{r_{+}^{2}}{4 \theta}}\right), \\
X_{9}=e^{-\frac{r_{+}^{2}}{4 \theta}}\left(r_{+}^{6}+12 \theta r_{+}^{4}+96 \theta^{2} r_{+}^{2}+384 \theta^{3}-384 \theta^{3} e^{\frac{r_{+}^{2}}{4 \theta}}\right) .
\end{gathered}
$$

For even number of dimensions there are no closed analytical forms. So, with numerical calculation of Hawking temperature in arbitrary number of spacetime dimensions, we show the results in forthcoming figures. For simplicity, hereafter we set $\theta=1$ in numerical calculations.

One motivation toward production and detection of micro-black holes in collider tests is that their evaporation process is not so clear for us. The evaporation process for charged black hole in the framework of noncommutativity or the generalized uncertainty principle $[4,22]$ is usually arranged in two phases. In the former phase, the temperature of the black hole grows during its evaporation until it approaches to a maximum value which is wellknown to the Hawking phase. The latter phase is noncommutative or GUP phase where in the noncommutative framework the temperature suddenly falls down from Hawking phase maximum to zero [14] while in the GUP framework it reaches to a nonzero, UV cutoff case with a finite nonzero mass which is known as Planck size remnant [4]. Therefore, the evaporation process terminates when black hole mass reaches to a fundamental mass and a consequent missing energy of order $\mathrm{TeV}$. The basic idea of a remnant is introduced by arguments that to cure the information loss problem [3]. The formation of stable black hole remnants would provide fascinating new signatures which admit for the recognition of such a black hole remnant event at near-future collider or UHECR experiments. Since the black hole remnant carries a significant fraction of the total micro-black hole mass, an amount of energy will be spent by the black hole in the Hawking thermal radiation. When the evaporation process ends, the black hole mass is in the Planck scale, leaving a remnant and an effective missing energy can be observed by searching for events of order $\mathrm{TeV}$ missing energy. Also, charged black hole remnants could remain a firm ionizing path electrically in the LHC detectors, e.g. ALICE, ATLAS, and CMS, that this could let to recognize the black hole remnants.

As figure 6 shows, assuming the fundamental Plank mass to be $0.4 \mathrm{TeV}$, the Hawking temperature increases with increasing the number of spacetime dimensions. Moreover the black hole remnant in extra dimensions has smaller mass than 4-dimensional one. Therefore, assuming a small enough fundamental energy-scales we expect micro-black holes in higher-dimensional spacetime to be hotter, and with a smaller mass at the endpoint of evaporation than 4-dimensional spacetime. When the charge of black hole varies as is shown in figure 7 , increasing the charge leads to decreasing the black hole temperature in a bulk spacetime but main changes occurs on the 3-brane due to the fact that in LED 


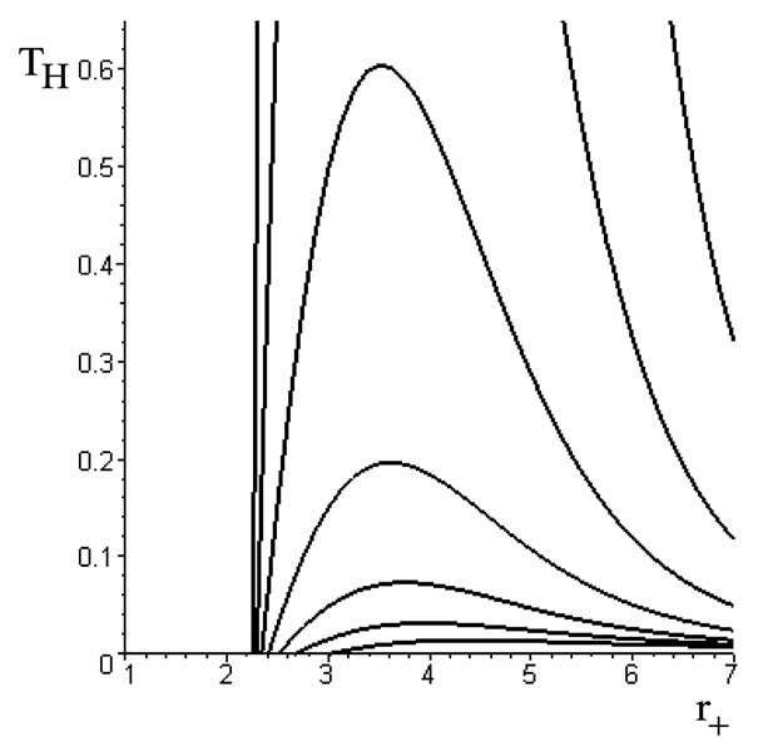

Figure 6: Black hole temperature, $T_{H}$, as a function of $r_{+}$for different number of spacetime dimensions. In this figure, black hole charge, mass and the $d$-dimensional Planck mass are set to be $Q=0.5, M=5$ and $M_{P l}=0.4$, respectively. On the right-hand side of the figure, curves are marked from bottom to top by $d=4$ to $d=10$. Figure shows that extra-dimensional black holes are hotter than four-dimensional black holes on the recognized regime.

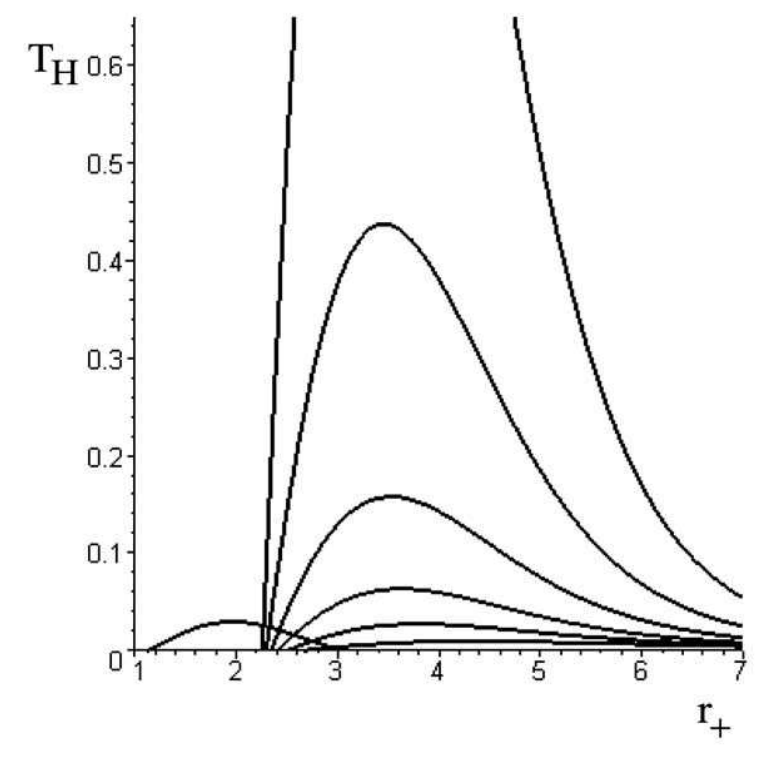

Figure 7: Black hole temperature, $T_{H}$, as a function of $r_{+}$for different number of spacetime dimensions. Black hole charge and mass and the $d$-dimensional Planck mass are set equal to $Q=2, M=5$ and $M_{P l}=0.4$ respectively. On the right-hand side of the figure, curves are marked from bottom to top by $d=4$ to $d=10$. The figure shows that, when the black hole charge varies main changes will be occurred on the brane (the short curve on the left-hand side of the figure). 


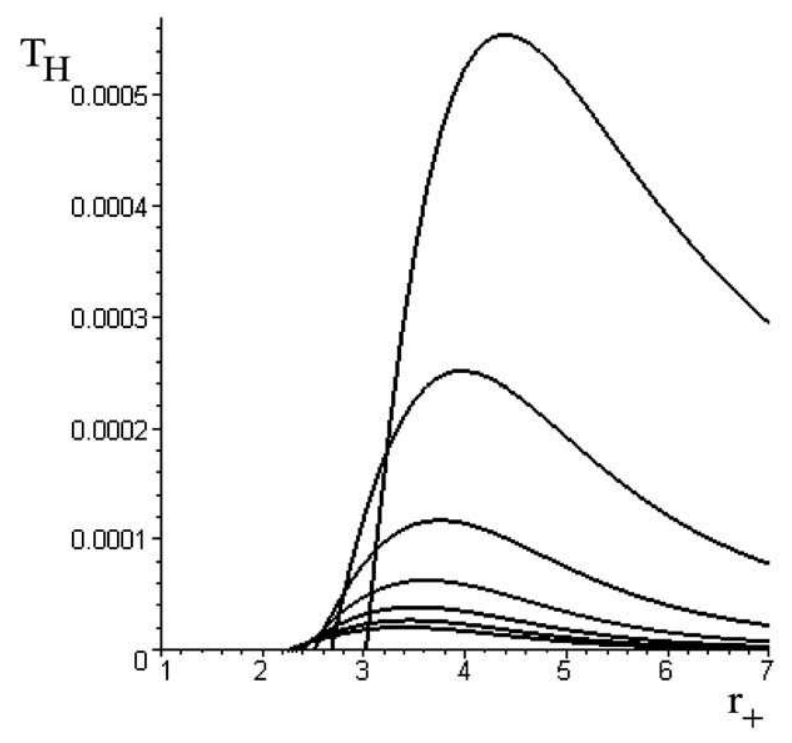

Figure 8: Black hole temperature, $T_{H}$, as a function of $r_{+}$for different number of spacetime dimensions. Black hole charge and mass and the $d$-dimensional Planck mass are set equal to $Q=0.5, M=5$ and $M_{P l}=2$ respectively. On the right-hand side of the figure, curves are marked from top to bottom by $d=4$ to $d=10$. The figure shows that with this value of $M_{P l}$, contrary to figure 6 , the extra-dimensional black holes are colder than four-dimensional black holes on the recognized regime.

scenarios, all standard-model particles are limited to our observable 3-brane, whereas gravitons can propagate the whole $d$-dimensional bulk substantially. As Emparan et al have shown, the main energy during Hawking radiation process from a $d$-dimensional black hole is emitted within modes on the brane because there are a great number of brane modes for standard model particles. Therefore, the main energy is expected to be radiated on the brane but there is only one graviton mode in the extra dimensions which can be propagated in the bulk [18]. Moreover, the numerical result for $d=4$ shows that no black hole is formed on the brane in this region. Eventually, in figure 8, by choosing $M_{P l}=2 \mathrm{TeV}$, we see that Hawking temperature decreases with increasing the number of spacetime dimensions, however black hole remnants masses will be smaller than 4-dimensional counterpart as shown in previous figures. Therefore, we expect microblack holes in higher-dimensional spacetime with a large fundamental energy-scale to be colder, and again with a smaller mass remnant than 4-dimensional counterpart. Our inspection has shown that for $M_{P l}=1.155 \mathrm{TeV}$, maximum Hawking temperature of black hole for $d=10$ is approximately equal to Hawking temperature of $d=4$ black hole. For $M_{P l}>1.155 \mathrm{TeV}$ and $d \leq 10$, black holes in extra dimensions are colder. Table 1 shows these results. As a general result, if large extra dimensions do really exist and the $d$-dimensional Planck mass to be less than $1 T e V$, a great number of black holes can be produced and detected in near-future colliders.

As another important thermodynamical properties, our next step is to calculate and investigate status of entropy variations in such a $d$-dimensional $\mathrm{RN}$-dS black hole. This 
Table 1: Comparison between black hole maximum temperature in four and extra spacetime dimensions for different values of $M_{P l}$.

\begin{tabular}{|l|l|}
\hline & $Q=0.5$ and $M=5 \mathrm{TeV}$ \\
\hline$M_{P l}=0.911 \mathrm{TeV}$ & $\left.\left.T_{H}(\max )\right|_{d=4} \approx T_{H}(\max )\right|_{d=5}$ \\
\hline$M_{P l}=0.915 \mathrm{TeV}$ & $\left.\left.T_{H}(\max )\right|_{d=4} \approx T_{H}(\max )\right|_{d=6}$ \\
\hline$M_{P l}=0.966 \mathrm{TeV}$ & $\left.\left.T_{H}(\max )\right|_{d=4} \approx T_{H}(\max )\right|_{d=7}$ \\
\hline$M_{P l}=1.026 \mathrm{TeV}$ & $\left.\left.T_{H}(\max )\right|_{d=4} \approx T_{H}(\max )\right|_{d=8}$ \\
\hline$M_{P l}=1.091 \mathrm{TeV}$ & $\left.\left.T_{H}(\max )\right|_{d=4} \approx T_{H}(\max )\right|_{d=9}$ \\
\hline$M_{P l}=1.155 \mathrm{TeV}$ & $\left.\left.T_{H}(\max )\right|_{d=4} \approx T_{H}(\max )\right|_{d=10}$ \\
\hline
\end{tabular}

entropy is defined as

$$
S=\int_{r_{0}}^{r_{+}} d r T_{H}^{-1} \frac{\partial M}{\partial r}
$$

where we find $S=0$ for the minimum horizon radius, $r=r_{0}$ (where black hole mass is minimized), which is a reasonable choice. A numerical evaluation of this expression for $M_{P l}=2$ is shown in figure 9 . The existence of the remnants is again approved from the thermodynamical behavior of such a system.

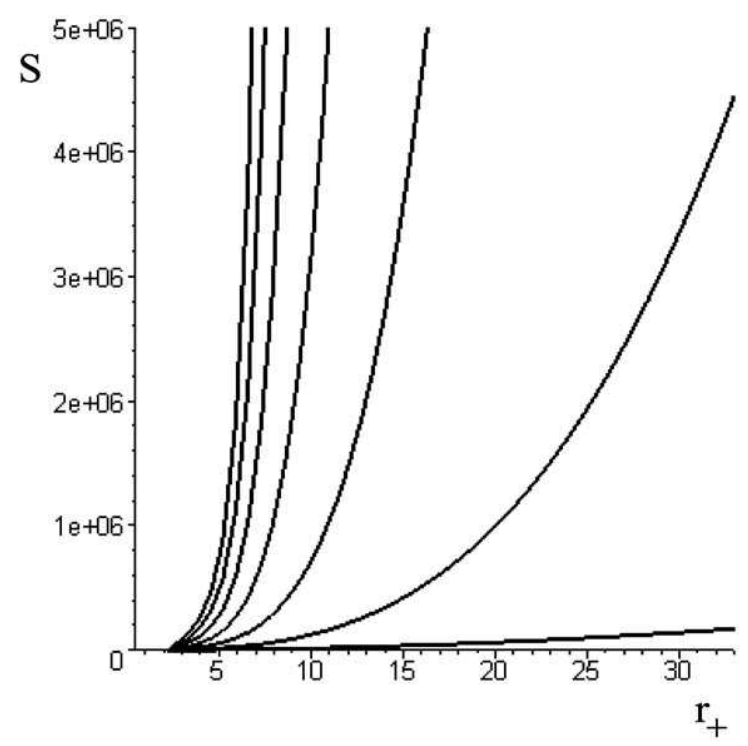

Figure 9: Black hole entropy, $S$, as a function of $r_{+}$for different number of spacetime dimensions. Black hole charge and the $d$-dimensional Planck mass are set equal to $Q=0.5$ and $M_{P l}=2$ respectively. On the right-hand side of the figure, curves are marked from bottom to top by $d=4$ to $d=10$. This figure shows that entropy increases with increasing the number of extra dimensions. Note that this result is depended on the value of $M_{P l}$. For smaller values of $M_{P l}$, the result is completely different.

Because of unusual thermodynamical properties of $\mathrm{TeV}$ black holes in noncommutative scenarios, it is interesting to investigate further thermodynamical details of these 
quantum gravity system. We first study the heat capacity of the black hole which can be obtained using the following relation

$$
C=\frac{\partial M}{\partial r_{+}}\left(\frac{\partial T_{H}}{\partial r_{+}}\right)^{-1} .
$$

The numerical results for $M_{P l}=2$ is presented in figure 10. This figure shows that black hole has a negative heat capacity (it means that $\left(\frac{\partial T_{H}}{\partial r_{+}}\right)^{-1}<0$ therefore $C<0$ in the Hawking phase) with a singularity for fixed values of $Q, M_{P l}$ and $d$. In fact, when the temperature reaches a maximum value of its amount where the slope of temperature curve $\left(\frac{\partial T_{H}}{\partial r_{+}}\right)^{-1}=0$ for a special $r_{+}$value, then the heat capacity becomes singular for this special value of $r_{+}$. For lower $r_{+}$, the temperature falls down (it means that $\left(\frac{\partial T_{H}}{\partial r_{+}}\right)^{-1}>0$ gives $C>0$ in noncommutative or GUP phase) to zero with a finite nonzero horizon radius, $r_{0}$ (which means that $C=0$ for the final stage of black hole evaporation).

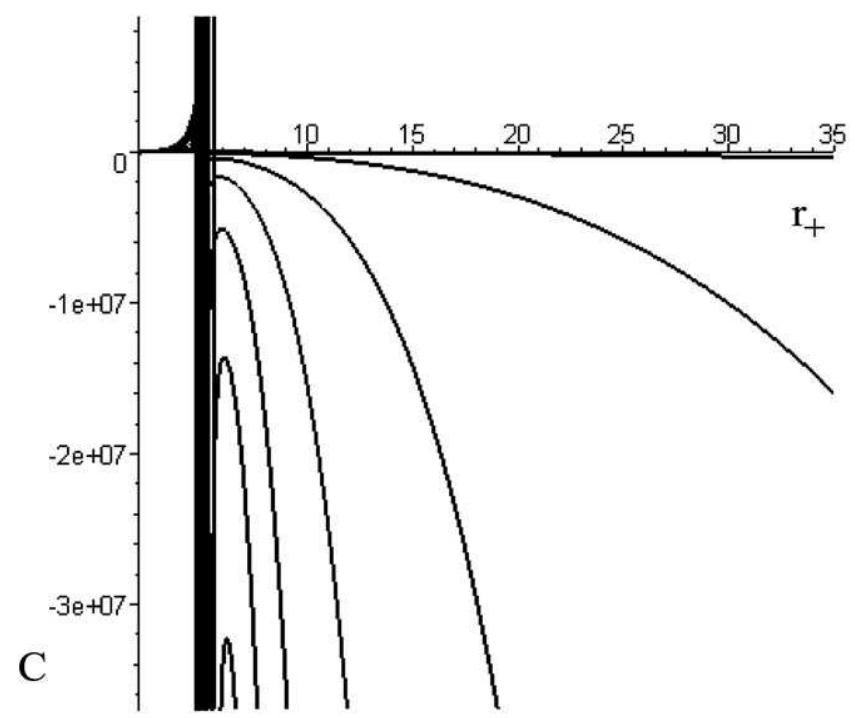

Figure 10: Black hole heat capacity, $C$, as a function of $r_{+}$, for different number of spacetime dimensions. Charge and the $d$-dimensional Planck mass are set equal to $Q=0.5$ and $M_{P l}=2$ respectively. On the right-hand side of the figure, curves are marked from top to bottom by $d=4$ to $d=10$.

Eventually, as a final remark in this section we consider the free energy of the RN-dS black hole that can be defined as

$$
F=M-T_{H} S
$$

The numerical calculations of this quantity are presented in figures 11 and 12. Reduction of the horizon size with decreasing free energy and approaching negative values of free energy for large values of $d$, can be seen both in these figures and in the equation (28). It is evident that for $r=r_{0}$, the free energy becomes equal to the minimum mass, $M_{0}$, 


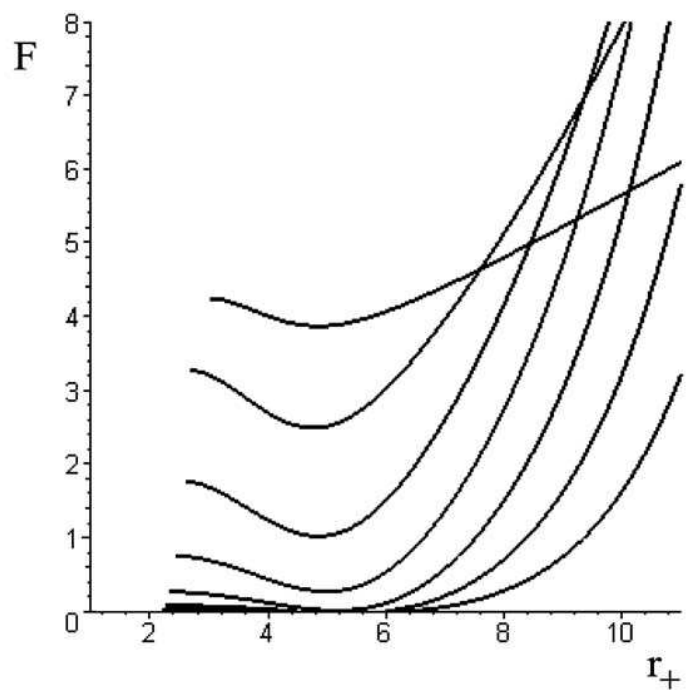

Figure 11: Black hole free energy, $F$, as a function of $r_{+}$, for different number of spacetime dimensions. Black hole charge and the $d$-dimensional Planck mass are set equal to $Q=0.5$ and $M_{P l}=0.4$ respectively. On the left-hand side of the figure, curves are marked from top to bottom by $d=4$ to $d=10$. As is evident, the behavior of free energy in our 3 -brane is very different to other dimensions due to maximum effects of charge on the 3-brane. The cutoff in the left hand side of the figure shows the existence of remnant.

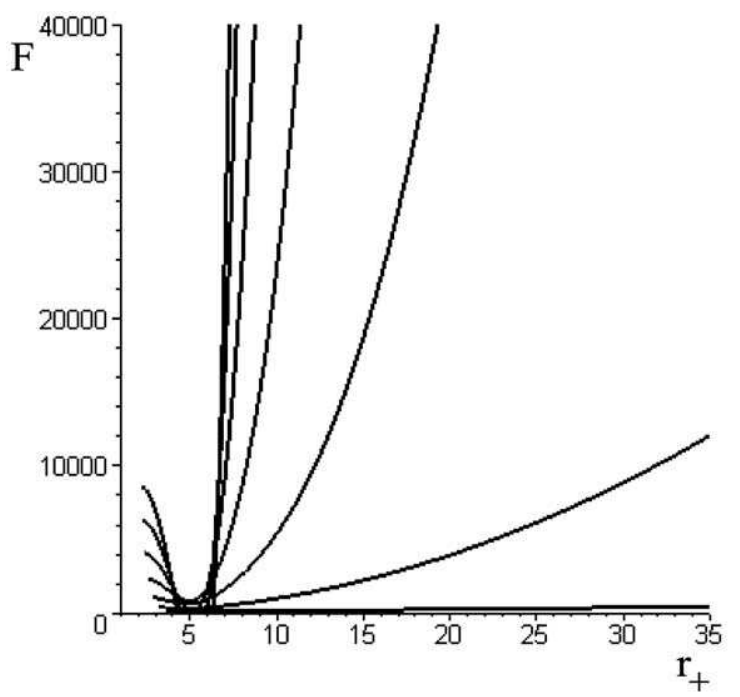

Figure 12: Black hole free energy, $F$, as a function of $r_{+}$, for different number of spacetime dimensions. Black hole charge and the $d$-dimensional Planck mass are chosen to be $Q=0.5$ and $M_{P l}=2$ respectively. On the right-hand side of the figure, curves are marked from bottom to top by $d=4$ to $d=10$. Contrary to the previous figure, here we see that increasing number of extra dimensions will increase the free energy of the system for this value of $M_{P l}$. The situation for small values of $M_{P l}$ is different as is shown in figure 11 . 
due to the fact that temperature or entropy are zero at this smeared-size, and therefore remnant is left over.

The idea of black hole remnant can cure both the singularity problem at the endpoint of black hole evaporation and information loss problem ${ }^{\S}$. In fact, if a stable black hole remnant is really exists due to the fact that there are some exact continuous global symmetries in the nature [24], then the minimum energy for black hole formation in collisions will be increased [25] (but depending on the number of extra dimensions). In this situation, the possibility of the production and detection of $T e V$-scale black holes may be decreased because of lowering the cross section for the expected LHC-energies and the absence of the final decay particles for the detection in the LHC detectors, e.g. ALICE, ATLAS, and CMS [26]. Therefore the idea of black hole remnant is most meaningful for us. Of course, it is important to note that if we consider the thermodynamic behavior at the very short distances (mass scales smaller than minimal mass) then it would be seen some exotic behavior of such a system. In a recent paper [27] we have reported some results about extraordinary thermodynamical behavior for Planck size black hole evaporation which may reflect the need for a fractal nonextensive thermodynamics [28] for Planck size black hole evaporation process. We just have shown that if nothing halts the evaporation process, the noncommutative black hole will throughout disappear eventually. However, in this case one encounters some unusual thermodynamical features leading to negative entropy, negative temperature and anomalous heat capacity where the mass of the black hole becomes of the order of Planck mass or less. There are two possible reasons for these unusual features: either we really cannot trust the details of the noncommutative effects with the Gaussian, Lorentzian and some other profiles of the smeared mass distribution at the regions that the mass of the black hole to be of the order of Planck mass [9] (see also [29] and [30]), or we really should doubt the results of standard thermodynamics at quantum gravity level which the origin of this premise may possibly becomes due to the fractal nature of spacetime at very short distances [27]. Indeed, at present we don't know which of these ideas are true.

\section{Summary and Discussion}

The noncommutative version of quantum field theories based on Moyal $\star$-product [31] lead to failure in resolving of some important problems, such as Lorentz invariance breaking, loss of unitarity and UV divergences of quantum field theory. Unfortunately, no flawless

\footnotetext{
${ }^{\S}$ Recently, we have shown that the form of the amendments for Hawking radiation as back-reaction effects with incorporation of GUP influences can recover the information. In this situation, correlations between the different modes of radiation evolve, which reflect the fact that at least part of the information leaks out from the black hole as the non-thermal GUP correlations within the Hawking radiation [23].
} 
and completely convincing theory of noncommutativity yet exists. However, the authors in Ref. [16] explained that the coordinate coherent states approach as a fascinating model of noncommutativity can be free from the problems mentioned above. In this approach, General Relativity in its usual commutative manner as described by the Einstein-Hilbert action remains applicable inasmuch, if noncommutativity effects can be treated in a perturbative manner, then this is defensible, at least to a good approximation. Indeed, the authors in Ref. [32] have shown that the leading noncommutativity corrections to the form of the Einstein-Hilbert action are at least second order in the noncommutativity parameter $\theta$. The generalization of the quantum field theory by noncommutativity based on coordinate coherent state formalism is also interestingly curing the short distance behavior of pointlike structures. Therefore, noncommutativity brings prominent qualitative and quantitative changes to the properties of black hole thermodynamics. Indeed, these changes could have important concepts for the possible formation and detection of black holes at the expected LHC-energies. In this paper, we have generalized the ANSS model of noncommutative Reissner-Nordström like geometries to model universes with large extra dimensions. Noncommutativity eliminates spacetime singularity due to smeared picture of particle mass and charge. The energy scales for production and detection of black holes remnants at LHC are examined and it has been shown that in the presence of noncommutativity, thermodynamical properties of $\mathrm{TeV}$ black holes depend on the values of fundamental Planck mass in extra dimensions. The possibility of black hole formation is reduced by increasing the charge of black hole particularly for 4-dimensional black hole on the brane. Since the center of mass energy of the proton-proton collision at LHC is $14 \mathrm{TeV}$, black hole formation is possible for $M_{\min }<14 \mathrm{TeV}$. Our analysis shows that if the number of spacetime dimension, $d$, increases with a larger amount of $d$-dimensional fundamental Planck mass, then the minimum energy for black hole formation in collisions will increase and we will not see any black hole at the usual $\mathrm{TeV}$ energy scales at LHC. In contrast, a smaller amount of $d$-dimensional fundamental Planck mass leads to conclusion that the minimum energy for black hole formation in collisions will decrease with increasing the number of extra dimensions and we are able to see black hole at the usual $T e V$ energy scales at the LHC. We have obtained an effective and noncommutative inspired cosmological constant in $d$-dimension which is leading to a finite curvature in the origin. From thermodynamics point of view, for a suitable choice of fundamental mass scale, Hawking temperature increases with increasing the number of spacetime dimensions. Moreover, the black hole remnant in extra dimensions has smaller mass than 4-dimensional one. Assuming a small enough fundamental energy-scales we expect microblack holes in higher-dimensional spacetime to be hotter, and with a smaller mass at the endpoint of evaporation than 4-dimensional spacetime. When the charge of black hole 
varies, increasing the charge leads to decreasing the black hole temperature in a bulk spacetime but main changes occurs on the 3-brane due to the fact that in LEDs scenarios, all standard-model particles are limited to our observable 3-brane, whereas gravitons can propagate the whole $d$-dimensional bulk substantially. The situation for the case with higher fundamental mass scale is different; in this situation the extra-dimensional black holes are colder than four-dimensional black holes on the recognized regime. Our analysis on $\mathrm{TeV}$ black hole production at the LHC shows that if large extra dimensions do really exist and the $d$-dimensional Planck mass to be less than $1 \mathrm{TeV}$, a great number of black holes can be produced and detected in LHC and other near-future colliders.

As a remark we accentuate that some authors have presented the black hole thermodynamics in the noncommutative framework adapting a coordinate noncommutativity against coherent state approach (see [8] and references therein). A question then appears: what is the difference between these two approaches? The standard way to handle the noncommutative problems is through the utilize of Moyal $\star$-product. That means to use complex number commuting coordinates and shift noncommutativity in the product between functions. This is mathematically valid, but it is physically useless since any model written in terms of $\star$-product, even the simplest field theory, is nonlocal and it is not obvious how to handle nonlocal quantum field theory. One suggested approach is perturbation in the $\theta$ parameter [33]. This is physically reasonable due to the fact that once expanded up to a given order in $\theta$, the resulting field theory becomes local. The smeared picture of particles based on coordinate coherent states defines complex number coordinates as quantum mean values of the original noncommuting ones between coordinate coherent states. In other words, in this setup one can see commuting coordinates as classical limit (in the quantum mechanical sense) of the noncommuting ones. In this framework, the emergent semiclassical geometry keeps memory of its origin. For example, free propagation of a point-like object is described by a minimal width Gaussian wave-packet as has been considered in our setup. So, the difference between two approaches lies in the definition of quantum field theoretical propagators.

\section{Acknowledgment}

This work has been supported partially by Research Institute for Astronomy and Astrophysics of Maragha, Iran. 


\section{References}

[1] S. W. Hawking, Commun. Math. Phys. 43, 199 (1975).

[2] J.D. Bekenstein, Lett. Nuovo. Cim. 4, 737 (1972); Phys. Rev. D 7, 2333 (1973); Phys. Rev. D 9, 3292 (1974).

[3] D. N. Page, Phys. Rev. Lett. 44, 301 (1980); G. t'Hooft, Nucl. Phys. B 256, 727 (1985); A. Mikovic, Phys. Lett. B 304 , 70 (1992); E. Verlinde and H. Verlinde, Nucl. Phys. B 406, 43 (1993); L. Susskind, L. Thorlacius and J. Uglum, Phys. Rev. D 48, 3743 (1993); D. N. Page, Phys. Rev. Lett. 71, 3743 (1993); Y. Aharonov, A. Casher and S. Nussinov, Phys. Lett. B 191, 51 (1987); T. Banks, A. Dabholkar, M. R. Douglas and M. OLoughlin, Phys. Rev. D 45, 3607 (1992), [arXiv:hep-th/9201061]; T. Banks and M. OLoughlin, Phys. Rev. D 47, 540 (1993), [arXiv:hep-th/9206055]; T. Banks, M. OLoughlin and A. Strominger, Phys. Rev. D 47, 4476 (1993), [arXiv:hepth/9211030]; S. B. Giddings, Phys. Rev. D 49, 947 (1994), [arXiv:hep-th/9304027].

[4] R. J. Adler, P. Chen and D. I. Santiago, Gen. Rel. Grav. 33, 2101 (2001), [arXiv:grqc/0106080].

[5] P. Nicolini, A. Smailagic and E. Spallucci, Phys. Lett. B 632, 547 (2006), [arXiv:grqc/0510112].

[6] H. S. Snyder, Quantized Spacetime, Phys. Rev. 71, 38 (1947); N. Seiberg and E. Witten, JHEP 9909, 032 (1999); M. R. Douglas and N. A. Nekrasov, Rev. Mod. Phys. 73, 977 (2001).

[7] F. Nasseri, Int. J. Mod. Phys. D 15, 1113 (2006), [arXiv:hep-th/0508122].

[8] K. Nozari and B. Fazlpour, Mod. Phys. Lett. A 22, 2917 (2007), [arXiv:hepth/0605109]; Acta Physica Polonica 39, 1363 (2008).

[9] T. G. Rizzo, J. High Energy Phys. (JHEP) 0609, 021 (2006), [arXiv:hepph/0606051]; E. Spallucci, A. Smailagic and P. Nicolini, Phys. Lett. B 670, 449 (2009), [arXiv:0801.3519]; P. Nicolini, Int. J. Mod. Phys. A 24, 1229 (2009), [arXiv:0807.1939].

[10] N. Arkani-Hamed, S. Dimopoulos and G. Dvali, Phys. Lett. B 429, 263 (1998), [arXiv:hep-ph/9803315]; N. Arkani-Hamed, S. Dimopoulos and G. Dvali, Phys. Rev. D 59, 086004 (1999), [arXiv:hep-ph/9807344]; I. Antoniadis, N. Arkani-Hamed, S. Dimopoulos and G. Dvali, Phys. Lett. B 436, 257 (1998), [arXiv:hep-ph/ 9804398]. 
[11] L. Randall and R. Sundrum, Phys. Rev. Lett. 83, 4690 (1999), [arXiv:hepth/9906064]; L. Randall and R. Sundrum, Phys. Rev. Lett. 83, 3370 (1999), [arXiv:hep-ph/9905221].

[12] G. Dvali, G. Gabadadze and M. Porrati, Phys. Lett. B 485, 208 (2000), [arXiv:hepth/0005016].

[13] P.C. Argyres, S. Dimopoulos, and J. March-Russell, Phys. Lett. B 441, 96 (1998); T. Banks and W. Fischler, [arXiv:hep-th/9906038]; R. Emparan, G. T. Horowitz and R. C. Myers Phys. Rev. Lett. 85, 499 (2000); S. Dimopoulos and G. Landsberg Phys. Rev. Lett. 87, 161602 (2001); S. B. Giddings and S. Thomas, Phys. Rev. D 65, 056010 (2002); M. B. Voloshin, Phys. Lett. B 518, 137 (2001); Phys. Lett. B 524, 376 (2002); S. N. Solodukhin, Phys. Lett. B 533, 153 (2002); G. T. Horowitz and J. Polchinski, Phys. Rev. D 66, 103512 (2002); V. Cardoso, E. Berti and M. Cavaglià, Class. Quant. Grav. 22, L61 (2005), [arXiv:hep-ph/0505125]; P. Kanti, Int. J. Mod. Phys. A 19, 4899 (2004); G. Landsberg, [arXiv:hep-ph/0211043]; M. Cavaglià, Int. J. Mod. Phys. A 18, 1843 (2003).

[14] S. Ansoldi, P. Nicolini, A. Smailagic and E. Spallucci, Phys. Lett. B 645, 261 (2007), [arXiv:gr-qc/0612035].

[15] S. B. Giddings and S. Thomas, Phys. Rev. D 65, 056010 (2002), [hep-ph/0106219].

[16] A. Smailagic and E. Spallucci, J. Phys. A 37, 7169 (2004), [hep-th/0406174]; J. Phys. A 36, L517 (2003), [arXiv:hep-th/0308193]; J. Phys. A 36, L467 (2003), [arXiv:hepth/0307217].

[17] J. L. Feng and A. D. Shapere, Phys. Rev. Lett. 88, 021303 (2002), [arXiv:hepph/0109106]; A. Ringwald and H. Tu, Phys. Lett. B 525, 135 (2002), [arXiv:hepph/0111042]; S. I. Dutta, M. H. Reno and I. Sarcevic, Phys. Rev. D 66, 033002 (2002), [arXiv:hep-ph/0204218]; R. Emparan, M. Masip and R. Rattazzi, Phys. Rev. D 65, 064023 (2002), [arXiv:hep-ph/0109287]; P. Jain, S. Kar, S. Panda and J. P. Ralston, Int. J. Mod. Phys. D 12, 1593 (2003), [arXiv:hep-ph/0201232]; M. Cavaglià, Int. J. Mod. Phys. A 18, 1843 (2003), [arXiv:hep-ph/0210296].

[18] R. Emparan, G. T. Horowitz and R. C. Myers, Phys. Rev. Lett. 85, 499 (2000), [arXiv:hep-th/0003118].

[19] P. Nicolini, J. Phys. A 38, L631 (2005), [arXiv:hep-th/0507266]; A. Gruppuso, J. Phys. A 38, 2039 (2005), [arXiv:hep-th/0502144]. 
[20] N. D. Birrell and P. C. W. Davies, Quantum Fields in Curved Space, Cambridge University Press; Reprint edition (April 27, 1984).

[21] R. M. Wald, Quantum Field Theory in Curved Spacetime and Black Hole Thermodynamics, University of Chicago Press, (1994).

[22] K. Nozari and S. H. Mehdipour, Mod. Phys. Lett. A 20, 2937 (2005), [arXiv:0809.3144]; K. Nozari and S. H. Mehdipour, Int. J. Mod. Phys. A 21, 4979 (2006), [arXiv:gr-qc/0511110]; A. J. M. Medved and E. C. Vagenas, Phys. Rev. D 70, 124021 (2004), [arXiv:hep-th/0411022].

[23] K. Nozari and S. H. Mehdipour, Europhys. Lett. 84, 20008 (2008), [arXiv:0804.4221].

[24] J. D. Bekenstein, Phys. Rev. D 5, 1239 (1972).

[25] M. Cavaglià, S. Das and R. Maartens, Class. Quant. Grav. 20, L205 (2003), [arXiv:hep-ph/0305223]; V. Cardoso, M. Cavaglià, L. Gualtieri, Phys. Rev. Lett. 96, 071301 (2006), [arXiv:hep-th/0512002]; S. Hossenfelder, In 'Focus on Black Hole Research', pp. 155-192, Nova Science Publishers (2005), [arXiv:hep-ph/0412265].

[26] B. Koch, M. Bleicher and S. Hossenfelder, J. High Energy Phys. (JHEP) 0510, 053 (2005), [arXiv:hep-ph/0507138].

[27] K. Nozari and S. H. Mehdipour, Chaos, Solitons and Fractals 39, 956 (2009), [arXiv:hep-th/0610076].

[28] C. Tsallis, Braz. J. Phys. 29, 1 (1999); C. Tsallis and E. Brigatti, Continuum Mech. Thermodyn. 16, 223 (2004).

[29] K. Nozari and S. H. Mehdipour, Class. Quant. Grav. 25, 175015 (2008), [arXiv:0801.4074].

[30] K. Nozari and S. H. Mehdipour, J. High Energy Phys. (JHEP) 03, 061 (2009), [arXiv:0902.1945].

[31] J. E. Moyal, Proc. Cambridge Phil. Soc. 45, 99 (1949).

[32] X. Calmet and A. Kobakhidze, Phys. Rev. D 72, 045010 (2005), [arXiv:hepth/0506157]; Phys. Rev. D 74, 047702 (2006), [arXiv:hep-th/0605275]; A. H. Chamseddine, Phys. Lett. B 504, 33 (2001), [arXiv:hep-th/0009153]; P. Aschieri et al, Class. Quant. Grav. 22, 3511 (2005), [arXiv:hep-th/0504183].

[33] M. Chaichian, M. M. Sheikh-Jabbari and A. Tureanu, Phys. Rev. Lett. 86, 2716 (2001), [arXiv:hep-th/0010175]. 